\section{Kidney \\ Blood Pressure Research}

\title{
Physical Activity in Solid Organ Transplant Recipients: Preliminary Results of the Italian Project
}

\author{
Giovanni Mosconi $^{a} \quad$ Vania Cuna ${ }^{b} \quad$ Maddalena Toniolib Valentina Tottic \\ Giulio S. Roi ${ }^{d}$ Patrizio Sarto ${ }^{e}$ Sergio Stefoni ${ }^{b}$ Manuela Trerotola ${ }^{f}$ \\ Alessandro Nanni Costa ${ }^{f}$
}

aOperative Unit of Nephrology and Dialysis, Morgagni Pierantoni Hospital, Forli; ${ }^{b} N e p h r o l o g y$, Dialysis, Renal Transplantation Unit, S. Orsola-Malpighi University Hospital, Bologna; ${ }^{~}$ No Profit Foundation for the Advancement of Organ and Tissue Transplantation (FITOT), Padova; ${ }^{d}$ Educational and Research Department Isokinetic Medical Group, Bologna; eUOC of Sport Medicine, ULSS Company 9, Treviso; Italian National Transplant Centre, Istituto Superiore di Sanità, Roma, Italy

\section{Key Words}

Kidney transplant - Liver transplant - Physical exercise - Muscle strength - Aerobic activity • Lifestyle $\cdot$ Cardio-vascular disease prevention

\begin{abstract}
Background/Aims: The role of physical activity in transplanted patients is often underestimated. We discuss the Italian National Transplant Centre experience, which started in 2008 studying transplanted patients involved in sports activities. The study was then developed through a model of cooperation between surgeons, sports physicians and exercise specialists. Methods: A multicentre study was realized in 120 transplanted patients of which 60 treated with supervised physical activity (three sessions/week of aerobic and strengthening exercises) and 60 controls. We present the results of the first 26 patients (16 males, 10 females; $47.8 \pm 10.0$ years; 21 kidney, 5 liver transplanted; time from transplant $2.3 \pm 1.4$ years) who completed 12 months of supervised physical activity. Results: Data showed an increase of peak aerobic power $(\mathrm{t}=4.535 ; \mathrm{P}<0.01)$ and maximum workload $(\mathrm{t}=4.665 ; \mathrm{P}<0.01)$ in the incremental cycling test. Maximum strength of knee extensors $(t=2.933 ; P<0.05)$ and elbow flexors $(t=2.450$; $\mathrm{P}<0.05)$, and the power of lower limb $(t=2.303 ; \mathrm{P}<0.05)$ significantly increases. Health Related Quality of Life showed a significant improvement. Serum creatinine $(1.4 \pm 0.5$ vs $1.3 \pm 0.4 \mathrm{mg} /$ $\mathrm{dL})$ and proteinuria $(0.10 \pm 0.14$ vs $0.08 \pm 0.08 \mathrm{gr} / \mathrm{dL})$ were stable. Conclusion: These preliminary results confirm the positive effects of supervised physical exercise. It can be considered as an input to promote other detailed exercise protocols.
\end{abstract}




\section{Kidney Blood Pressure Research}

\section{Introduction}

Physical inactivity is an important risk factor for cardio-vascular disease: it was recently defined as a pandemic threat with repercussions on health care [1]. Several studies report positive effects of physical exercise in the prevention of primary and secondary cardiovascular disease [2-3]; other papers stress the role of physical activity in the management of chronic diseases [4], in the improvement of the quality of life and relieving depression and anxiety symptoms. In recent years the importance of which type of exercise should be administered has emerged. The association of aerobic and strengthening exercises seems to have a synergic action on physical fitness and on metabolism regulation; this last effect occurs through a cross talk between skeletal muscle and adipose tissue [5-7]. The most recent data show that, especially in risk categories, programs of focused intervention to facilitate the practicing of regular physical activity are advantageous [8].

The population with solid transplanted organs is characterized by a high risk of development of cardio-vascular disease [9-10]. Insulin resistance is a frequent feature of transplanted patients; the development of metabolic syndrome is usually associated to a chronic inflammation sub-clinic status and a sarcopenia [11-12]. Immunosuppressive therapy promotes the development of dysmetabolism and the worsening of sarcopenia [1315]. Despite these physiopathologic and epidemiologic premises, the role of physical activity is largely underestimated in Transplant Centres. Transplanted patients have low levels of exercise capacity and are also frequently physically inactive [16-17]. The knowledge of the transplant world is still far from a regular and concrete application of physical activity as a possibility of prevention; there is a lack of awareness regarding this problem and hesitation on behalf of the patients and their families. Furthermore, the practice of regular exercise has psychological and organizational limitations. In this population of patients, there are only papers on limited clinical records and conducted with different methods for short periods; at present, the positive effect of physical activity is not well supported on long term outcomes such as mortality and graft survival [18-20]. In relation to this encouraging participation of physical activity for transplanted recipients, since 2008, the Italian National Transplant Centre has set up the "Transplant ...and now Sport" project to investigate and promote the diffusion of physical activity and sports in transplanted recipients.

\section{Italian Experience}

"Transplant ... and now Sport" Project. The Project, called as "Transplant ... and now Sport", started by the idea of considering physical activity as a non pharmacological therapy, was developed by means of a model of cooperation between medical transplantation doctors, sports physicians and exercise specialists (graduates in Physical Education and physiotherapists); some patient associations (such as Italian Association of Dialyzed and Transplanted Patients ANED) actively adhered to the project. Initially the project consisted in clinical, physiological and psychological evaluations (cardio-circulatory response to physical stress, energy expenditure, Health-Related Quality of Life) of a group of solid organ transplanted patients participating in the Italian National Championship (alpine skiing, track \& field events, cycling, swimming, volleyball and trekking); it was highlighted that transplant recipients involved in sports competitions are able to attain a level of daily physical activity consistent with a healthy lifestyle [21]. The performances are often comparable to that of people practicing recreational physical activity and depend on the level of weekly training, as in the general population $[17,22,23]$. In transplanted patients practicing amateur physical activity during an observation period of 2-5 years, no significant changes of the kidney function were recorded; in this population a higher level of well-being was perceived than that recorded in inactive ones and comparable with the general population [24].

Some interesting research was carried out to study the changes in renal function expressed as serum creatinine and proteinuria after intense physical exercise (cycling marathon of $130 \mathrm{~km}$ with a total climb of $1871 \mathrm{~m}$ ) in transplanted patients compared with 


\section{Kidney \\ Blood Pressure Research}

Fig. 1. Tasks of several of the components involved in the research protocol "Trapianto...and now Sport". The professionals are in close contact with each other.

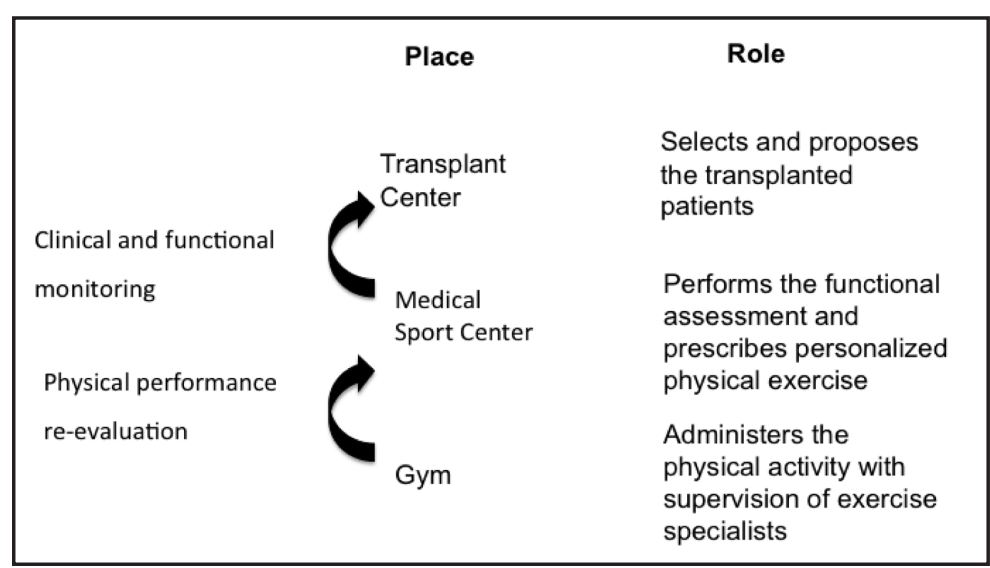

a group of amateur cyclists; the results showed a similar trend in the two analyzed groups (increase of creatinine and proteinuria at the end of the excercise) and a rapid return to baseline values (within 18-24 hours); in transplanted patients a reduced capacity of urine concentration was observed, compatible with tubular damage (personal observation).

As a result of these findings (safety during physical activity, improvement of the psychological status), the National Transplant Centre has created a research protocol with the aim of verifying whether prescribed physical activity for transplant recipients is able to improve both the biological parameters and the physical fitness with positive effects on graft survival. This project also aims to promote a detailed diffusion of physical activity in the transplanted population. The study was started in two Italian regions (Emilia-Romagna and Veneto) and progressively expanded to another 7 regions (Toscana, Piemonte, Abruzzo, Molise, Sicilia, Lazio, Lombardia) and to the Autonomous Region of Bolzano.

The purpose was to create a network where Transplant Centres select patients, the Sports physicians prescribe personalised physical exercise on the basis of performance in functional tests, and graduates in physical education and physiotherapists administer and supervise the prescribed physical activity (Figure 1). All the physicians, the physiotherapists and graduates in Physical Education involved in the study must participate in a one day course, aimed to implement and to share their knowledge on the clinical aspects of transplanted recipients, on the effects of physical exercise, and on the protocol of the study and the methods of training. By means of 7 specific courses, 38 transplantation doctors, 84 sports physicians and 133 exercise specialists were trained. In this paper preliminary results are given regarding the patients who completed one year of supervised physical activity.

\section{Materials and Methods}

The "Transplant ...and now Sport" protocol is a multicentre controlled, prospective, non-randomized study that considers the enrolment of 120 patients (range 18-60 years), with solid organ (kidney, liver, heart) transplantation (range 6 months to 8 years of life transplantation), with clinical and functional stabilities checked by the Transplant Centres. The duration of the observation period for each patient is 12 months. Patients are divided into two groups: 60 patients (Group A) in which personalized physical activity is prescribed by the Sport physicians, 60 control patients (Group B) in which some generic lifestyle indications are given without specific prescription. The enrolment in group B was due to logistical problems (distance from the Medical Sport Centre, impossibility of patients to go to the gym). Written informed consent is obtained from the patients before inclusion, according to the procedures approved by the Ethical Committee of Transplant Centre S. Orsola-Malpighi Hospital, Bologna, Italy. Exclusion criteria are: orthopaedic limitations, psychiatric or neurological disorders, rejection episodes or graft functional variations in the last 6 months and any other cardiovascular contraindication to physical exercise or training. Clinical status and functional evaluation of the organ graft, blood levels (renal function, lipid and glucidic status, electrolytic 


\section{Kidney \\ Blood Pressure Research}

balance, hepatic function, blood count), urinalysis, body composition (anthropometric measurement, bioimpedence analysis) and cardiovascular assessments are performed by the Transplantation Centre to assess the exclusion criteria. The SF-36 questionnaire is used to evaluate self-reported domains of health status; that includes eight scales of Health-Related Quality of Life (HRQoL): Physical Functioning (PF), Role Limitation due to Physical Health (RP), Bodily Pain (BP), General Health (GH), Vitality (VT), Social Functioning (SF), Role Limitations due to Emotional Health (RE) and Mental Health (MH). These scales have a score from 0 to 100, with a higher score being more positive.

The Medical Sport Centre carries out the functional assessment tests for exercise capacity and muscle strength in both groups. Incremental cycling test is performed on a cycle ergometer starting from 20W, with increments of $20 \mathrm{~W}$ every 4 minutes until voluntary exhaustion, the appearance of electrocardiographic signs of pathology or inappropriate blood pressure response occur. A 12-lead electrocardiogram is monitored continuously throughout the test. At each step blood pressure is measured and a capillary blood sample from the earlobe is taken to measure blood lactate concentration, in order to estimate the workload corresponding to aerobic (S2) and anaerobic (S4) thresholds, conventionally declared at 2 and $4 \mathrm{mM}$ of lactate, respectively.

Oxygen uptake $\left(\mathrm{V}^{\prime} \mathrm{O}_{2}\right)$ is determined continuously using an open circuit method (Sensor Medics Corp., Anaheim, CA, USA), calibrated against known gas mixtures before each test. Oxygen uptake at the aerobic and anaerobic thresholds in relation with the workload (W) and heart rate (HR) are then calculated. Oxygen uptake at the highest tolerated level of exercise is also determined $\left(\mathrm{V}^{\prime} \mathrm{O}_{2 \text { peak }}\right)$.

Muscle strength of some muscular groups in the lower (knee extensors, plantar flexors), and upper (elbow flexors, elbow extensors and shoulder abductors) limbs was calculated by the one repetition maximum (1RM) strength tests. The power of the lower limbs is measured indirectly from the fly time of a countermovement jump (CMJ) (Optojump, Microgate, Italy).

On the basis of the tests, the Sports physicians prescribe the individual programme of supervised exercise (aerobic and strengthening exercises) for the patients in group A. These patients are sent to an approved Gym to start the prescribed physical activity under the supervision of a suitably trained physiotherapist or a graduate in Physical Education with a frequency of three times per week. The aerobic exercise is performed on a stationary bike (30 minutes), with heart rate corresponding to the aerobic threshold, previously determined. The right intensity is continuously monitored by heart rate monitors (Polar, Finland), so the heart rate of the patient is maintained constant with eventual slight adjustments of the mechanical power of the stationary bike. Strengthening exercises consist in 2 sets of 20 repetitions at $35 \%$ of the previously determined $1 \mathrm{RM}$, for each of the selected muscle groups of the upper and lower limbs. For the patients in group B the physicians recommend generic exercises for physical fitness without prescription as usual routine patient management. Patients of both groups are checked at baseline $\left(\mathrm{T}_{0}\right)$, returning back to the Transplantation and to the Medical Sport Centres at 6 months $\left(\mathrm{T}_{6}\right)$ and at 12 months $\left(\mathrm{T}_{12}\right)$ from the time of enrolment to repeat both the clinical and the functional assessment tests performed at $\mathrm{T}_{0}$.

Statistical methods

Descriptive statistics (means \pm standard deviations) were calculated for all continuous variables. Paired t-test was used to evaluate the differences between $\mathrm{T}_{0}$ and $\mathrm{T}_{12}$ for each variable, applying post-hoc Bonferroni analysis (SPSS statistical program). Statistical significance was set at $\mathrm{P}<0.05$.

\section{Results}

In Preliminary: One hundred and fifty transplanted recipients have been considered between September 2010 and September 2013. Thirty-eight recipients were excluded from the Transplant Centres due to cardiovascular problems that needed further investigation, functional limitations or inadequate compliance. At present, 112 patients are enrolled (74 and 38 in Group A and Group B respectively). Therefore, this preliminary analysis is based on 26 patients from group A (16 males, 10 females, mean age $47.8 \pm 10.0$ years, 21 kidney and 5 liver transplanted, mean time from transplant of 2.3 


\section{Kidney Blood Pressure Research}

1.4 years) that have already reached 12 months since the enrolment date. The number of drop-outs recorded was 5 in the supervised group (road traffic accident 1, sports injury unrelated to physical supervised exercise 1, lack of motivation 2 , gastro-intestinal problems 1).

The Body Mass Index (BMI) didn't change after 12 months $\left(\mathrm{T}_{0} 24.2 \pm 3.6\right.$; $\mathrm{T}_{6} 24.2 \pm 3.7$; $\mathrm{T}_{12} 23.9 \pm 3.9 \mathrm{~kg} / \mathrm{m}^{2}, \mathrm{P}>0.05$ ). However the percentage of fat mass tends to decrease after 6 months of supervised physical activity that continued until the 12 months follow-up $\left(\mathrm{T}_{0} 21.1 \pm 8.3 ; \mathrm{T}_{6} 20.3 \pm 7.0 ; \mathrm{T}_{12} 19.9 \pm 6.8 \%, \mathrm{P}>0.05\right)$.

In the incremental cycling test for estimating maximal oxygen uptake the maximum workload was significantly increased at $\mathrm{T}_{6}$ and remained stable at $\mathrm{T}_{12}\left(\mathrm{~T}_{0} 92 \pm 33 ; \mathrm{T}_{6} 112 \pm 39\right.$; $\left.\mathrm{T}_{12} 109 \pm 41 \mathrm{~W} ; \mathrm{t}=4.665 ; \mathrm{P}<0.05\right)$. The maximal oxygen uptake $\left(\mathrm{V}^{\prime} \mathrm{O}_{2 \text { peak }}\right)$ in relation to $\mathrm{HR}$ max also significantly increased after the 6 months follow-up and continued to increase in $\mathrm{T}_{12}\left(\mathrm{~T}_{0} 22.0 \pm 6.9 ; \mathrm{T}_{6} 24.6 \pm 7.4 ; \mathrm{T}_{12} 25.3 \pm 7.3 \mathrm{~mL} / \mathrm{kg} / \mathrm{min} ; \mathrm{t}=4.535 ; \mathrm{P}<0.05 ; \mathrm{T}_{0} 135 \pm 23 ; \mathrm{T}_{6} 138 \pm 24\right.$; $\mathrm{T}_{12} 142 \pm 26$ bpm t=3.101; $\mathrm{P}<0.05$ ).

In relation to the maximum strength of lower limbs we found a significant increase in the maximum strength of knee extensor (dominant limb) at $\mathrm{T}_{6}$ that continued to increase until $\mathrm{T}_{12}\left(\mathrm{~T}_{0} 93 \pm 33 ; \mathrm{T}_{6} 104 \pm 38, \mathrm{~T}_{12} 107 \pm 44 \mathrm{~kg}, \mathrm{t}=2.933 ; \mathrm{P}<0.05\right)$. In the counter-movement jump test we found a significant increase in the flight time $(\mathrm{s})$ and in the jump height $(\mathrm{cm})$ at $\mathrm{T}_{6}$ that continued to increase at $\mathrm{T}_{12}\left(\mathrm{~T}_{0} 0.424 \pm 0.122 ; \mathrm{T}_{6} 0.435 \pm 0.119 \mathrm{~T}_{12} 0.443 \pm 0.118 \mathrm{~s}\right.$; $\left.\mathrm{t}=2.846 \mathrm{P}<0.05 ; \mathrm{T}_{0} 22.9 \pm 11.5 ; \mathrm{T}_{6} 24.8 \pm 10.0 ; \mathrm{T}_{12} 25.7 \pm 10.0 \mathrm{~cm} ; \mathrm{t}=2.303, \mathrm{P}<0.05\right)$. The increase in power in the upper limbs was of less significant. The maximum strength of elbow flexors (dominant limb) increased at $\mathrm{T}_{6}$ and remained stable at $\mathrm{T}_{12}\left(\mathrm{~T}_{0} 8.9 \pm 2.7 ; \mathrm{T}_{6} 10.3 \pm 2.6\right.$; $\mathrm{T}_{12}$ 10.4 $\pm 3.4 \mathrm{~kg} \mathrm{t}=2.450 ; \mathrm{P}<0.05$ ).

No episodes of acute rejection were recorded. Serum creatinine was stable between the beginning and end of the observation period $(1.4 \pm 0.5 \mathrm{mg} / \mathrm{dL}$ vs. $1.3 \pm 0.4 \mathrm{mg} / \mathrm{dl}$ and $0.10 \pm 0.14 \mathrm{~g} / \mathrm{dL}$ vs $0.08 \pm 0.08 \mathrm{~g} / \mathrm{dL}$ respectively). We have not experienced any increase in proteinuria $(0.10 \pm 0: 14 \mathrm{~g} / \mathrm{dL}$ vs $0.08 \pm 0.08)$. In SF-36 questionnaire all the physiological variables increased at T12; in particular, the improvement in the perception of body pain $\left(\mathrm{T}_{0} 72 \pm 33 ; \mathrm{T}_{6} 74 \pm 29 ; \mathrm{T}_{12} 81 \pm 24 ; \mathrm{t}=2.274\right)$, general health $\left(\mathrm{T}_{0} 58 \pm 25 ; \mathrm{T}_{6} 66 \pm 23 ; \mathrm{T}_{12} 71 \pm 22\right.$; $\mathrm{t}=2.118)$, vitality $\left(\mathrm{T}_{0} 61 \pm 24 ; \mathrm{T}_{6} 65 \pm 24 ; \mathrm{T}_{12} 72 \pm 20 ; \mathrm{t}=2.513\right)$, social functioning $\left(\mathrm{T}_{0} 67 \pm 24\right.$; $\mathrm{T}_{6} 74 \pm 28 ; \mathrm{T}_{12} 84 \pm 19 ; \mathrm{t}=2.883$ ), and role-emotional $\left(\mathrm{T}_{0} 76 \pm 33 ; \mathrm{T}_{6} 92 \pm 19 ; \mathrm{T}_{12} 94 \pm 18 ; \mathrm{t}=2.526\right.$ ) was statistically significant $(\mathrm{P}<0.05)$.

\section{Discussion}

The importance of therapeutic and preventive physical activity is underestimated in the follow-up of patients undergoing solid organ transplantation; transplant centres have a low propensity to suggest physical exercise. To counteract the concerns of clinicians and the fears of patients and family (risk of injuring the transplanted graft, protective attitude) further data and clinical evidence is needed. The project "Transplant ...and now Sport" is an attempt to overcome these barriers (insufficient knowledge of the characteristics of transplanted patients, poor inter-professional coordination, lack of information given on the benefits of exercise, lack of post-transplant logistics), which can limit the spread of the practice of physical exercise in this patient population. The long time experience carried out by the Italian National Transplant Centre in the transplanted patients that practises regular amateur sport activity and physical supervised exercise is widely positive in terms of safety with beneficial effects on the perception of quality of life. The study, in which we present preliminary data, as far as we know, is the first analysing a long period of supervised physical activity (1 year) with a "personalised prescription" on the basis of the performance tests reached at the beginning of the study. Enrolment selection criteria (age, time from transplant $>6$ months - 8 years) makes the case records sufficiently homogeneous. One strong point is that the association of aerobic and strengthening exercises are consistent with the more 


\section{Kidney Blood Pressure Research}

recent physiopathology acquirements [7]; the complementary action of these two types of exercise can lead to a recovery of the skeletal muscle anabolism with positive metabolic effects.

Prescribed tests are easily performed and management criteria are not subjective (for aerobic exercise S2 and S4, for strengthening exercises 35\% of 1 RM). Physical exercise in the gym was managed only by properly trained personnel; the centralization of the monitoring study has allowed for consistent management of the Project. Currently, safety was recorded for the prescribed physical activity according to the proposed model; no interruptions of the protocol were registered for problems relating to the physical activity. A significant number of non suitable patients (38/150), selected from the Transplant Centres, emerged; it is possible that the first experience of physical activity prescription in structures that are not included in the health field in coordination with Medical Sport Centres has limited the enrolment of patients with cardio-vascular disease or functional limitations; the experience gained will extend to the possibility of supervised physical activity for a wider range of patients. A low number of drop outs ( 5 out of $74 ; 7 \%$ ) was positively affected by selection criteria and care during the follow up period; this suggests that the supervision of the physical activity by motivated professionals plays an important role in the adherence to the treatment.

On the whole, the preliminary data confirm the results of previous papers regarding the importance of supervised and prolonged physical activity [19-20] though the limited number of patients who completed the follow-up doesn't allow for definitive conclusions.

These preliminary results show a significant increase in aerobic fitness (maximum oxygen uptake, adaptation to the workload); similarly, improvements $>15 \%$ in the muscular leg strength. Variations in the strengthening of the arms $(<3 \%)$ are not in line with these results. One possible explanation is that the presence of arterio-venous fistula in patients with transplanted kidney limited the intensity of the arm muscle exercises programmed during the study.

Even in the presence of reduced percentage variations, preliminary data confirm the improvement of the nutritional status in patients practising regular physical activity, as recently reported [25]. The trend to reduce the fat mass is promising for the prevention of metabolic syndrome; the secretion of inflammatory adipokines has been shown as a mechanism to induce peripheral tissue insulin resistance [4-26]. The study of the changes induced by physical activity on the inflammatory state of the patient transplanted constitutes a field of particular interest for the future.

Regular physical activity according to the proposed protocol does not interfere negatively on renal function and proteinuria. The data are in line with the long-term experience of our group about the trend of renal function in transplanted patients who regularly practice sport activities and about the physiological responses to acute stress such as a cycling marathon of $130 \mathrm{~km}$ (personal observation).

The improvement of HRQoL confirms the importance of physical exercise in the management of chronic diseases [16-27]; in our experience, the practice of amateur sport activity can further improve the HRQoL [24]. There are some limitations in this preliminary report. Firstly we do not present or discuss the results of the control group. A preliminary analysis not showed in this group significant improvements in the physiological and psychological parameters, but until now the number of control patients who completed the observation period is too small to make an adequate comparison.

A second limitation is represented by the non-randomized design that is dependent on the adopted model in order to allow the selected patients to practise physical activity in gyms with trained professionals close to their homes. To date, not all the different areas involved have the same opportunities. The accessibility to the facilities where patients can easily carry out the supervised programmes of exercise seems to be crucial in every proposal of exercise as a therapy; the surplus value of the project was the creation in Italy of a network between Transplant Centres, Medical Sport Centres and Graduates in Physical Education/Physiotherapy, which will be able in the future to cooperate in the management 


\section{Kidney \\ Blood Pressure Research}

of the transplanted patients ( $>250$ educated professionals). We would like that this interdisciplinary cooperation to be used also for patients waiting for transplantation [28].

On the basis of the preliminary results of this study we can affirm that an appropriate dose of physical training can represent a useful, safe and non-pharmacologic contribution to the treatment of transplanted patients with significant improvements of physiological variables related to physical fitness (cardiovascular and musculoskeletal systems, perceived HRQoL). The sustainability, also economic, should be evaluated on a wider scale. Although it remains appropriate to avoid over medicalization of exercise, it is important to remember that physical activity is a preventive measure with a lower economic impact compared to pharmacological therapy; actually, among the other hypothesis, we suggest initial periods of supervised exercise before giving autonomous personalised therapy at home. Randomized trials with prolonged periods of observation are necessary to assess the impact of regular physical activity on morbidity/mortality of patients and survival of the graft.

\section{Conclusion}

The level of rehabilitation in transplanted patients allows them to resume physical and sports activities with positive effects on aerobic metabolism, muscle strength and HRQoL. Preliminary results of the study protocol "Transplant ...and now Sport" confirm the efficacy of the model based on the cooperation between Transplantation Centres, Medical Sport Centres and Gyms in the administration of supervised exercise. Despite some limitations, this paper can be considered an input to discuss, develop and promote other detailed exercise protocols for transplant recipients and to foster improved post transplantation health and survival. Furthermore, we think that the preliminary data of our study can assist transplant professionals in counselling transplant recipients thus helping to ensure that physical activity becomes a safe routine medical treatment plan of patient management. We would like the concept to be expanded, if possible, to the patients waiting for transplantation.

\section{Disclosure Statement}

The authors of this work declare that they do not have any conflict of interests.

\section{References}

$>1$ Kohl HW $3^{\text {rd }}$, Craig CL, Lambert EV, Inoue S, Alkandari JR, Leetongin G, Kahlmeier S, Lancet Physical Activity Series Working Group: The pandemic of physical inactivity: global action for public health. Lancet 2012;380:294-305.

2 Myers J, Prakash, M, Froelicher V, Do D, Partington S, Atwood JE: Exercise capacity and mortality among men referred for exercise testing. N Engl J Med 2002;346:793-801.

- 3 Leitzmann MF, Park Y, Blair A, Ballard-Barbash R, Mouw T, Hollenbeck AR, Schatzkin A: Physical activity recommendations and decreased risk of mortality. Arch Intern Med 107;167:2453-2460.

4 Gleeson M, Bishop NC, Stensel DJ, Lindley MR, Mastana SS, Nimmo MA: The anti-inflammatory effects of exercise: mechanisms and implications for the prevention and treatment of disease. Nat Rev Immunol 2011;11:607-615.

-5 LeBrasseur NK, Walsh K, Arany Z: Metabolic benefits of resistance training and fast glycolytic skeletal muscle. Am J Physiol Endocrinol Metab 2011;300:E3-E10.

6 Aoi W, Naito Y, Yoshikawa T: Dietary exercise as a novel strategy for the prevention and treatment of metabolic syndrome: effects on skeletal muscle function. J Nutr Metab 2011;2011:676208.

7 Perez-Terzic CM: Exercise in cardiovascular diseases. PM R 2012;4:867-873. 


\section{Kidney \\ Blood Pressure Research}

-8 Heath GW, Parra DC, Sarmiento OL, Andersen LB, Owen N, Goenka S, Montes F, Brownson RC: Evidence based intervention in physical activity: lessons from around the world. Lancet 2012;380:271-281.

-9jo AO: Cardiovascular complications after renal transplantation and their prevention. Transplantation 2006;82:603-611.

10 Pilmore H, Dent H, Chang S, McDonald SP, Chadban SJ: Reduction in cardiovascular death after kidney transplantation. Transplantation 2010;89:851-857.

-11 Sharif A: Metabolic Syndrome and Solid-Organ Transplantation. Am J Transplant 2010;10:12-17.

12 Fahal IH: Uraemic sarcopenia: aetiology and implications. Nephrol Dial Transplant 2013, DOI 10.1093/ndt/ gft070.

13 Dickinson JM, Drummond MJ, Fry CS, Gundermann DM, Walker DK, Timmerman KL, Volpi E, Rasmussen BB: Rapamycin administration in humans blocks the contraction-induced increase in skeletal muscle protein synthesis. J Physiol 2009;587:1535-1546.

14 Ma K, Mallidis C, Bhasin S, Mahabadi V, Artaza J, Gonzalez-Cadavid N, Arias J, Salehian B: Glucocorticoidinduced skeletal muscle atrophy is associated with upregulation of myostatin gene expression. Am J Physiol Endocrinol Metab 2003;285:E363-E371.

15 Sakuma K, Yamaguchi A: The functional role of calcineurin in hypertrophy, regeneration, and disorders of skeletal muscle. J Biomed Biotechnol 2010;2010:721219.

16 Painter P, Marcus RL: Assessing physical function and physical activity in patients with CKD. Clin J Am Soc Nephrol 2013;8:861-872.

17 Mosconi G, Roi GS, Nanni Costa A, Stefoni S: Attività fisica nei pazienti con trapianto di rene. G Ital Nefrol 2011;28:174-187.

18 Macdonald JH, Kirkman D, Jibani M: Kidney transplantation: a systematic review of interventional and observational studies of physical activity on intermediate outcomes. Adv Chronic Kidney Dis 2009;16:482500.

19 Heiwe S, Jacobson SH: Exercise training for adults with chronic kidney disease. Cochrane Database Syst Rev 2011;10:CD003236.

20 Didsbury M, McGee RG, Tong A, Craig JC, Chapman JR, Chadban S, Wong G: Exercise training in solid organ transplant recipients: a systematic review and meta-analysis. Transplantation 2013;95:679-687.

21 Roi GS, Parigino M, Pisoni D, Mosconi G, Nanni Costa A, Stefoni S: Energy expenditure during a day of sport competitions in kidney transplant recipients. Transplantation 2010:90:1136-1138.

22 Mosconi G, Colombo D, Graziani E, Franceschelli N, Roi GS, Totti V, Nanni Costa A, Stefoni S: Physical performance in kidney transplanted patients: a study on desert trekking. J Biol Regul Homeost Agents 2011;25:417-425.

23 Roi GS, Mosconi G, Capelli I, Cuna V, Persici E, Parigino M, Pisoni D, Todeschini P, Costa AN, Stefoni S: Alpine skiing and anaerobic performance in solid organ transplant recipients. Transplant Proc 2010;42:10291031.

24 Mazzoni D, Cicognani E, Mosconi G, Totti V, Roi GS, Trerotola M, Nanni Costa A: Sport Activity and HealthRelated Quality of Life After Kidney Transplantation. Transplant Proc 2014 (accepted and in press).

-25 Zelle DM: The role of diet and physical activity in post-transplant weight gain after renal transplantation. Clin Transplant 2013;27:E484-E490.

-26 Kwon H, Pessin EJ: Adipokines mediate inflammation and insuline resistance. Front Endocrinol (Lausanne) 2013;4:71.

27 Gordon EJ, Prohaska T, Siminoff LA, Minich PJ, Sehgal AR: Needed: tailored exercise regimens fro kidney transplant recipients. Am J Kidney Dis 2005;45:769-774.

28 Rosas SE, Reese PP, Huan Y, Doria C, Cochetti PT, Doyle A: Pretransplant physical activity predicts all-cause mortality in kidney transplant recipients. Am J Nephrol 2012;35:17-23. 